\title{
On the Metric Dimension of Infinite Graphs*
}

\author{
J. Cáceres ${ }^{\dagger} \quad$ C. Hernando M. Mora $^{\ddagger} \quad$ I. M. Pelayo $\quad$ M. L. Puertas $\|$
}

November 8, 2018

\begin{abstract}
A set of vertices $S$ resolves a graph $G$ if every vertex is uniquely determined by its vector of distances to the vertices in $S$. The metric dimension of a graph $G$ is the minimum cardinality of a resolving set. In this paper we study the metric dimension of infinite graphs such that all its vertices have finite degree. We give necessary conditions for those graphs to have finite metric dimension and characterize infinite trees with finite metric dimension. We also establish some results about the metric dimension of the cartesian product of finite and infinite graphs, and give the metric dimension of the cartesian product of several families of graphs.
\end{abstract}

Key words: infinite graph, locally finite graph, resolving set, metric dimension, cartesian product

\section{Introduction}

Throughout this paper a graph $G$ is an ordered pair of disjoint sets $(V, E)$ where $V$ is nonempty and $E$ is a subset of unordered pairs of $V$. The vertices and edges of $G$ are the elements of $V=V(G)$ and $E=E(G)$ respectively. We say that a graph $G$ is finite (resp. infinite) if the set $V(G)$ is finite (resp. infinite). The degree of a vertex $u \in V(G)$ is the number of edges containing $u$ and is denoted by $\operatorname{deg}_{G}(u)$, or simply $\operatorname{deg}(u)$ if the graph $G$ is clear. An graph is locally finite if every vertex has finite degree. All the graphs considered in this paper are connected and locally finite.

Infinite graphs have been studied by many authors as D. König [9], C. St. J. A. NashWilliams [12], C. Thomassen [16], R. Diestel and many other authors [6].

We denote the distance between two vertices $u$ and $v$ by $d_{G}(u, v)$, or simply $d(u, v)$ if the graph $G$ is clear. A vertex $x$ in a graph $G$ resolves two vertices $u, v$ if $d(u, x) \neq d(v, x)$. A subset of vertices $S$ is a a resolving set of $G$ if for any two vertices, there exists a vertex in $S$ that resolves them. A resolving set with minimum cardinality is a metric basis. If a graph $G$ has at least a finite resolving set, the metric dimension $\beta(G)$ of $G$ is the cardinality of a metric basis, otherwise we say that the metric dimension of $G$ is infinite. If

\footnotetext{
*Research partially supported by projects JA FQM305, MTM2005-08441-C02-01, MEC-MTM200601267, DURSI2005SGR00692, MTM2005-08990-C02-01

${ }^{\dagger}$ Universidad de Almería, Almería, Spain, jcaceres@ual.es

‡Universitat Politècnica de Catalunya, Barcelona, Spain, carmen.hernando@upc.edu

${ }^{\S}$ Universitat Politècnica de Catalunya, Barcelona, Spain, merce.mora@upc.edu

『Universitat Politècnica de Catalunya, Barcelona, Spain, ignacio.m.pelayo@upc.edu

"Universidad de Almería, Almería, Spain, mpuertas@ual.es
} 
$S=\left\{x_{1}, \ldots, x_{n}\right\}$ is a finite set of vertices of $G$, we denote by $r(u \mid S)$ the vector of distances from $u$ to the vertices of $S$, that is, $r(u \mid S)=\left(d\left(u, x_{1}\right), \ldots, d\left(u, x_{n}\right)\right)$. Then, $S$ is a resolving set if and only if $r(u \mid S) \neq r(v \mid S)$ for all vertices $u \neq v$. If $S$ is a finite metric basis we say that $r(u \mid S)$ are the metric coordinates of vertex $u$ respect to $S$. A resolving set does not necessarily contain a metric basis. For example, any two distinct vertices of a path form a resolving set, but a metric basis of this graph contains exactly one of its endpoints. This fact makes more difficult to determine the metric dimension of a graph.

Resolving sets in general graphs were defined by Harary and Melter [11] and Slater [15]. Resolving sets have been widely investigated [5, 3, 7] and appear in many diverse areas including coin weighting [1, network discovery and verification [2, robot navigation [10, 14], connected joins in graphs [13], and strategies for the Mastermind game [8].

In this paper we study resolving sets in infinite graphs. The first question that arises is to determine infinite graphs with finite resolving sets. In Section 2 we give necessary conditions for this and in Section 3 we characterize acyclic connected infinite graphs, i.e. infinite trees, with finite metric dimension. On the other hand, interesting examples of graphs can be obtained as cartesian products of graphs. The metric dimension of cartesian products of finite graphs is studied in [3]. In Section 4 we give bounds for the metric dimension of cartesian products of finite and infinite graphs and determine the metric dimension for certain families of graphs.

\section{Finite Metric Dimension}

A natural starting point for studying the metric dimension of infinite graphs is to investigate the finiteness of this invariant. It is not difficult to realize that infinite graphs may have finite or infinite metric dimension. In fact, for every $k \geq 0$ there exist infinite graphs with metric dimension $k$. To prove this, we define the one-way infinite path, $P_{\infty}$, as the infinite graph with set of vertices $V\left(P_{\infty}\right)=\left\{u_{i}: i \geq 0\right\}$, and two vertices $u_{i}, u_{j}$ are adjacent if and only if $|i-j|=1$. We say that $u_{0}$ is the endpoint of $P_{\infty}$. In a similar way we define for $k \geq 2$ the $k$-way infinite path, $P_{k \infty}$, as the graph formed by $k$ pairwise disjoint one-way infinite paths and a new vertex adjacent to their $k$ endpoints. It is straightforward to prove that $\beta\left(P_{\infty}\right)=1, \beta\left(P_{2 \infty}\right)=2$ and $\beta\left(P_{k \infty}\right)=k-1$, if $k \geq 3$ (see Figure 11). It is also easy to verify that finite paths and the one-way infinite path are the only graphs with metric dimension equal to 1 , since there exists a vertex $v$ for which there is at most one vertex at distance $k$, for every $k \geq 0$.

Proposition 1. Then the metric dimension of a graph $G$ is 1 if, and only if, $G$ is either a finite path or the one-way infinite path.

So, the metric dimension of any infinite graph $G \neq P_{\infty}$ is at least 2. However, apart from $P_{2 \infty}$ and $P_{3 \infty}$, there are many more infinite graphs with metric dimension 2 as, for example, any graph obtained by attaching a finite path or a one-way infinite path at an arbitrary vertex of degree 2 of $P_{\infty}$. On the other hand, if we attach one leaf at every vertex of the one-way infinite path we obtain the so-called infinite comb graph, $B_{\infty}$, which satisfies $\beta\left(B_{\infty}\right)=\infty$ (see Figure 2).

Proposition 2. The infinite comb graph $B_{\infty}$ has infinite metric dimension.

Proof. Suppose that $V\left(B_{\infty}\right)=\left\{u_{i}: i \geq 0\right\} \cup\left\{v_{i}: i \geq 0\right\}$ and $E\left(B_{\infty}\right)=\left\{u_{i} u_{i+1}: i \geq\right.$ $0\} \cup\left\{u_{i} v_{i}: i \geq 0\right\}$. If $B_{\infty}$ has a finite resolving set $S$, there exists a vertex $u_{k}$ such that $u_{i}$, 

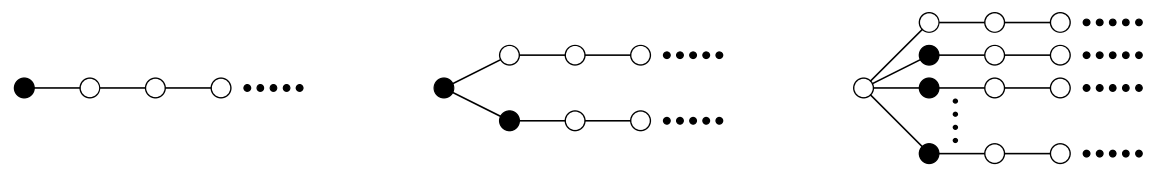

Figure 1: Black vertices form a metric basis of the graphs $P_{\infty}$ (left), $P_{2 \infty}$ (middle) and $P_{k \infty}$ (right).

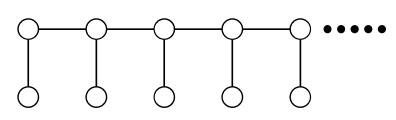

Figure 2: The infinite comb graph $B_{\infty}$ has infinite metric dimension.

$v_{i}$ are not in $S$ for all $i \geq k$. Then, $d\left(w, u_{k+1}\right)=d\left(w, u_{k}\right)+1=d\left(w, v_{k}\right)$ for every $w \in S$. Hence, $S$ does not resolve the pair $u_{k+1}$ and $v_{k}$.

In going on from finite to infinite graphs, a natural technique is to study the desired property or parameter for their induced subgraphs. Nevertheless, this seems to go nowhere in the case of the metric dimension. Let us illustrate this remark with some examples.

The infinite comb graph has infinite metric dimension, nevertheless the metric dimension of every finite induced subgraph is at most 2 .

The metric dimension of the graph in Figure 3 is 2, nevertheless it does contain induced subgraphs with infinite metric dimension, as for example the infinite comb graph, and induced subgraphs with metric dimension $k$, for every $k \geq 1$ (see Figure 3).
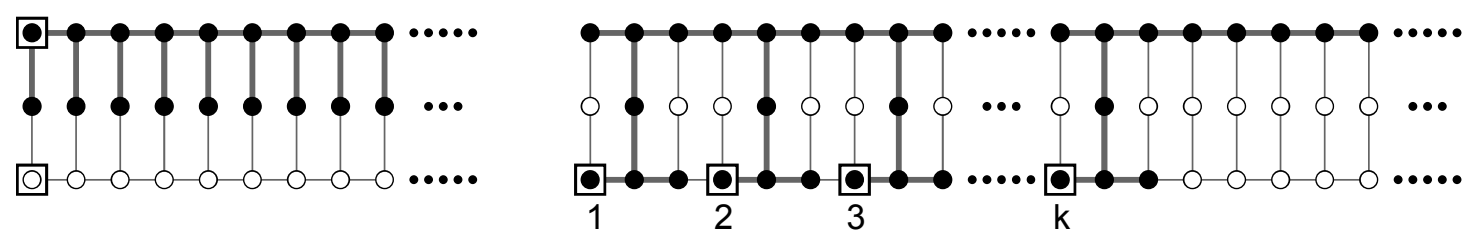

Figure 3: Left, squared vertices form a metric basis of the illustrated graph, while black vertices induce the graph $B_{\infty}$, with infinite metric dimension. Right, top vertices induce the graph $P_{\infty}$ with metric dimension 1 , and for every $k \geq 2$, squared vertices form a metric basis of the infinite subgraph induced by black vertices.

We conclude this section by obtaining two necessary conditions for an infinite graph to have finite metric dimension.

\subsection{Uniformly locally finite graphs}

An infinite graph is uniformly locally finite if there exists a positive integer $M$ such that the degree of every vertex is at most $M$. For example, the graphs $P_{k \infty}$ and $B_{\infty}$ are uniformly locally finite, and the graph obtained by hanging $i$ vertices of degree 1 to the vertex at distance $i \geq 0$ from the endpoint of $P_{k \infty}$ is non uniformly locally finite (see Figure 4). 


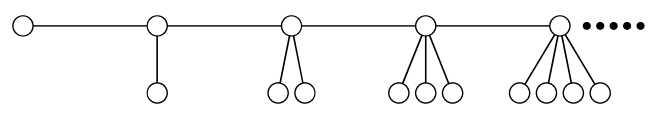

Figure 4: An example of non uniformly locally finite graph.

Lemma 1. If $G$ is an infinite graph with $\beta(G)=k$, then every vertex of $G$ has degree at most $3^{k}-1$.

Proof. Let $S=\left\{x_{1}, \ldots, x_{k}\right\}$ be a metric basis of $G$. Consider a vertex $v$ and its metric coordinates respect to $S, r(v \mid S)=\left(d\left(v, x_{1}\right), d\left(v, x_{2}\right), \ldots, d\left(v, x_{k}\right)\right)$. If $w$ is adjacent to $v$, then $r(v \mid S) \neq r(w \mid S)$ and $\left|d\left(v, x_{i}\right)-d\left(w, x_{i}\right)\right| \leq 1$ for all $x_{i} \in S$. This implies that there are only $3^{k}-1$ different possibilities for $r(w \mid S)$. Since all vertices must have distinct metric coordinates, the degree of $v$ is at most $3^{k}-1$.

As an immediate consequence of this lemma we get the following necessary condition for an infinite graph to have finite metric dimension.

Theorem 1. If $G$ is an infinite graph with finite metric dimension, then it is uniformly locally finite.

Note that the reciprocal of this result is not true, being the infinite comb graph illustrated in Figure 2 a counterexample.

\subsection{Disjoint metric rays}

A metric ray with endpoint $u_{0}$ in a graph $G$ is an infinite subgraph $P$ of $G$ isomorphic to the one-way infinite path, such that there exists an ordering of its vertex set, $V(P)=\left\{u_{0}, u_{1}, u_{2}, u_{3}, \ldots\right\}$, with $u_{k}$ adjacent to $u_{k+1}$ in $P$ for all $k \geq 0$, and $d_{G}\left(u_{0}, u_{k}\right)=$ $d_{P}\left(u_{0}, u_{k}\right)=k$.

Probably, the oldest and best known result about infinite graphs is the König's infinity Lemma (see [9]), that is equivalent to the following assertion.

Lemma 2. For every vertex $v$ of an infinite graph, there exists a metric ray with $v$ as endpoint.

Next, we show some results concerning metric rays which will help us to find a second necessary condition for an infinite graph to have finite metric dimension, quite different from that of Theorem 1 .

Lemma 3. Let $P$ be a metric ray of an infinite graph $G$ with ordered vertex set $V(P)=$ $\left\{u_{0}, u_{1}, u_{2}, \ldots\right\}$. Then, for every vertex $x$ of $G$ there always exists an integer $i_{0} \geq 0$ such that for every $j \geq i_{0}, d\left(u_{j+1}, x\right)=d\left(u_{j}, x\right)+1$.

Proof. Let $\left(a_{i}\right)_{i \geq 0}$ be the sequence defined as $a_{i}=d\left(u_{i}, x\right)+d\left(x, u_{0}\right)-i$. Since $P$ is a metric ray, we have that $d\left(u_{i}, x\right)+d\left(x, u_{0}\right) \geq d\left(u_{i}, u_{0}\right)=i$, hence $a_{i} \geq 0$. On the other hand, since $u_{i+1}$ and $u_{i}$ are adjacent vertices, we have $d\left(u_{i+1}, x\right) \leq d\left(u_{i}, x\right)+1$, and consequently $a_{i+1}=d\left(u_{i+1}, x\right)+d\left(x, u_{0}\right)-(i+1) \leq d\left(u_{i}, x\right)+1+d\left(x, u_{0}\right)-i-1=d\left(u_{i}, x\right)+d\left(x, u_{0}\right)-i=a_{i}$. Hence, $\left(a_{i}\right)_{i \geq 0}$ is a decreasing sequence of non-negative integer numbers. Therefore, there exists $i_{0}$ such that $a_{j+1}=a_{j}$ for all $j \geq i_{0}$, or equivalently $a_{j}=k$ for all $j \geq i_{0}$. But this implies that $d\left(u_{j+1}, x\right)=d\left(u_{j}, x\right)+1$ for all $j \geq i_{0}$. 
Lemma 4. Let $P$ be a metric ray of an infinite graph $G$, with ordered vertex set $V(P)=$ $\left\{u_{0}, u_{1}, u_{2}, \ldots\right\}$, and $S$ a finite subset of vertices of $G$. There always exists an integer $i_{0} \geq 0$ such that for every $k \geq 0, r\left(u_{i_{0}+k} \mid S\right)=r\left(u_{i_{0}} \mid S\right)+\underbrace{(k, \ldots, k)}_{|S|}$.

Proof. Let $S=\left\{x_{1}, \ldots, x_{n}\right\}$. By Lemma 3 , for every $h \in[1, n]$ there exists $i_{h}$ such that $d\left(u_{j+1}, x_{h}\right)=d\left(u_{j}, x_{h}\right)+1$ for all $j \geq i_{h}$. Let $i_{0}=\max \left\{i_{h}: h \in[1, n]\right\}$. Then $d\left(u_{j+1}, x_{h}\right)=$ $d\left(u_{j}, x_{h}\right)+1$ for all $j \geq i_{0}$ and $h \in[1, n]$. This implies that $d\left(u_{j+k}, x_{h}\right)=d\left(u_{j}, x_{h}\right)+k$ for all $k \geq 0, j \geq i_{0}$ and $h \in[1, n]$. In particular, $r\left(u_{i_{0}+k} \mid S\right)=r\left(u_{i_{0}} \mid S\right)+\underbrace{(k, \ldots, k)}_{|S|}$.

For every finite set $S$ of vertices and every metric ray $P$ in an infinite graph $G$, we denote by $i(P, S)$ the minimum among all those integers $i_{0}$ satisfying the conditions of the preceding lemma.

At this point, we recall the notion of doubly resolving set introduced in [3]. Two vertices $x$ and $y$ in a nontrivial graph $G$ doubly resolve a pair of vertices $u$ and $v$ if $d(u, x)-d(v, x) \neq$ $d(u, y)-d(v, y)$. If $S$ and $U$ are two subsets of vertices of $G$, we say that $S$ doubly resolves $U$ if every pair of distinct vertices in $U$ are doubly resolved by two vertices in $S$. We say that $S$ is a doubly resolving set of $G$ if $S$ doubly resolves $V(G)$. If $G$ has at least one finite doubly resolving set, we define $\psi(G)$ as the minimum cardinality of a doubly resolving set; otherwise, we say that $\psi(G)=\infty$. Observe that every doubly resolving set is also a resolving set, which means that $\beta(G) \leq \psi(G)$. Therefore, the next logical step should be to study the finiteness of this parameter.

Lemma 5. A finite set of vertices does not doubly resolve any infinite set of vertices of an infinite graph.

Proof. Suppose that $S=\left\{x_{1}, \ldots, x_{n}\right\}$ is a finite set and $U$ an infinite set of vertices of a graph $G$. Let $D=\max \left\{d\left(x_{i}, x_{j}\right): x_{i}, x_{j} \in S\right\}$. For every vertex $u \in U$, we define $f_{\left(x_{i}, x_{j}\right)}(u)=$ $d\left(u, x_{i}\right)-d\left(u, x_{j}\right) \in[-D, D]$, and $f_{S}(u)=\left(f_{p_{1}}(u), \ldots, f_{p_{m}}(u)\right)$, where $\left\{p_{1}, \ldots, p_{m}\right\}=$ $\left\{\left(x_{i}, x_{j}\right): x_{i}, x_{j} \in S, x_{i} \neq x_{j}\right\}, m=n(n-1)$. Since there are only $2 D+1$ possible values for $f_{p_{h}}(u)=f_{\left(x_{i}, x_{j}\right)}(u)$, we have that $\left|\left\{f_{S}(u): u \in U\right\}\right| \leq(2 D+1)^{n(n-1)}$. But $U$ in an infinite set, so there are at least two different vertices $u, v \in U$ such that $f_{S}(u)=f_{S}(v)$. This implies that for every pair $\left(x_{i}, x_{j}\right) \in S \times S, x_{i} \neq x_{j}$, we have $d\left(x_{i}, u\right)-d\left(x_{j}, u\right)=$ $f_{\left(x_{i}, x_{j}\right)}(u)=f_{\left(x_{i}, x_{j}\right)}(v)=d\left(x_{i}, v\right)-d\left(x_{j}, v\right)$. Then, $S$ does not doubly resolve $U$.

Corollary 1. If $G$ is an infinite graph, then $\psi(G)=\infty$.

Lemma 6. Let $G$ be an infinite graph and let $S$ be a finite resolving set of $G$. Suppose that $\mathcal{P}$ is a set of metric rays in $G$ with pairwise disjoint vertex sets. Then $S$ doubly resolves the set of vertices $\left\{u_{i(P, S)} \mid P \in \mathcal{P}\right\}$.

Proof. Suppose on the contrary that $S$ does not doubly resolve $W=\left\{u_{i(P, S)} \mid P \in \mathcal{P}\right\}$. Then, there exists a pair of distinct vertices $u, v \in W$ such that $d(x, u)-d(x, v)=d(y, u)-$ $d(y, v)$ for every pair of distint vertices $x, y \in S$. This implies that $d(x, u)-d(x, v)=k$ for every $x \in S$, and consequently $r(v \mid S)=r(u \mid S)+(k, \ldots, k)$. If $k \geq 0$, by Lemma 4 there exists a vertex $w$ in the same metric ray of $u$ such that $r(w \mid S)=r(u \mid S)+(k, \ldots, k)=$ $r(v \mid S)$, which contradicts the fact that $S$ is a resolving set of $G$. If $k<0$ we consider $d(x, v)-d(x, u)=-k>0$ and proceed in the same way. 
As a consequence of the preceding lemmas we obtain another necessary condition for infinite graphs having finite metric dimension.

Theorem 2. If $G$ is an infinite graph with finite metric dimension, then it does not contain an infinite number of metric rays with pairwise disjoint vertex sets.

The reciprocal of Theorem 2 is not true: it is easy to verify that the graph of Figure 5 has infinite metric dimension, however it contains at most two metric rays with pairwise disjoint vertex sets.

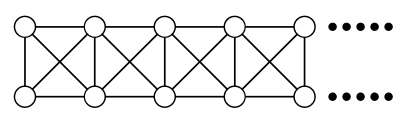

Figure 5: A graph with infinite metric dimension not containing infinite metric rays with pairwise disjoint vertex sets.

Note that the conditions stated in Theorems 1 and 2 are independent. For example, the graph in Figure 4 is a non-uniformly locally finite graph not containing infinite metric rays with pairwise disjoint vertex sets. On the other hand, the infinite grid, illustrated in Figure 6, is uniformly locally finite, but contains infinite metric rays with pairwise disjoint vertex sets. Observe also that both conditions together are not sufficient to assure finite metric dimension. For example, the infinite comb graph has infinite metric dimension and satisfies both conditions.

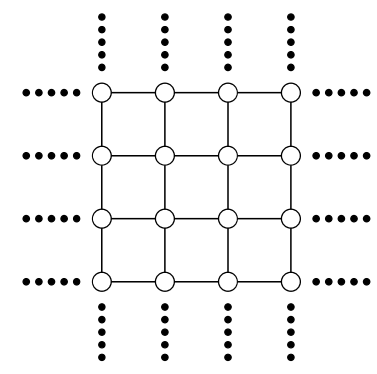

Figure 6: The infinite grid.

\section{Infinite Trees}

This section is devoted to study the metric dimension of infinite trees, that is, connected acyclic infinite graphs. We obtain a finiteness characterization and calculate the exact value of this parameter.

Theorem 3. An infinite tree has finite metric dimension if and only if the set of vertices of degree at least three is finite.

Proof. The only infinite trees without vertices of degree at least three are $P_{\infty}$ and $P_{2 \infty}$, which satisfy $\beta\left(P_{\infty}\right)=1$ and $\beta\left(P_{2 \infty}\right)=2$. Now assume that $T$ is an infinite tree with some vertex of degree at least three. 
$(\Rightarrow)$ Suppose first that $S$ is a finite resolving set for an infinite tree $T$. Consider the finite set $S^{*}$ formed by all vertices lying in a path with its endpoints in $S$. If there are infinite vertices with degree at least three, there exists a vertex $w \notin S^{*} \operatorname{such}$ that $\operatorname{deg}(w) \geq 3$. Since $S^{*}$ induces a connected subgraph in $T$ and $T$ is acyclic, there is only one vertex $z \in S^{*}$ such that $d(w, z)=\min \left\{d(w, x): x \in S^{*}\right\}$. Let $w^{\prime}$ be the vertex adjacent to $w$ that lies in the unique $w-z$ path. Since $\operatorname{deg}(w) \geq 3$, there are at least two vertices $u$, $v$ different from $w^{\prime}$ and adjacent to $w$, such that all paths to the vertices in $S \subseteq S^{*}$ pass through $w$. Hence, $r(u \mid S)=r(w \mid S)+(1, \ldots, 1)=r(v \mid S)$, contradicting the hypotheses that $S$ is a resolving set.

$(\Leftarrow)$ Now suppose that the set $W=\{x \in V: \operatorname{deg}(x) \geq 3\}$ is finite. Consider the finite set $S$ formed by all vertices lying in a path with endpoints in $W$ and all vertices adjacent to some vertex of $W$. We claim that $S$ is a resolving set for $T$. Since $S$ induces a connected subgraph in $T$ and $T$ is acyclic, for every vertex $u$ not in $S$ there is only one vertex $z(u) \in S$ such that $d(u, z(u))=\min \{d(u, x): x \in S\}$. By definition of $S, z(u) \notin W$. This implies that $\operatorname{deg}(z(u))=2$. Let $u, v$ be distinct vertices not in $S$ and consider the vertices $z(u), z(v) \in S$. If $z(u) \neq z(v)$, then there is only a path joining $u$ and $v$ that passes trough $z(u)$ and $z(v)$, and $z(u)$ or $z(v)$ resolves the pair $u, v$. If $z(u)=z(v)$, then $\{u, v, z(u)\}$ induces a path in $T$ with $z(u)$ as an endpoint. Hence, $z(u)$ resolves $u$ and $v$.

Let $v$ be a vertex of a (finite or infinite) tree $T$ with maximum degree at least three. A branch of $T$ at $v$ is a maximal subtree having $v$ as a leaf. A branch path of $T$ at $v$ is a branch that is either a finite path or a 1-way infinite path. Let $P_{T}(v)$ be the number of branch paths of $T$ at $v$ (see Figure 7). Observe that the number of branches of $T$ at $v$ is exactly the degree of $v$, and $P_{T}(v) \geq 2$ implies $\operatorname{deg}(v) \geq 3$. In [4, 10, 15] it was proved that the metric dimension of a finite tree different from a path is $\beta(T)=\sum_{\operatorname{deg}(v) \geq 3} \max \left\{P_{T}(v)-1,0\right\}$. It was also shown that every metric basis of $T$ is obtained by taking, for each vertex $v$ such that $P_{T}(v) \geq 2$, exactly $P_{T}(v)-1$ vertices different from $v$ lying on different branch paths of $T$ at $v$. Next we prove that the same results also hold for infinite trees (see Figure 8).
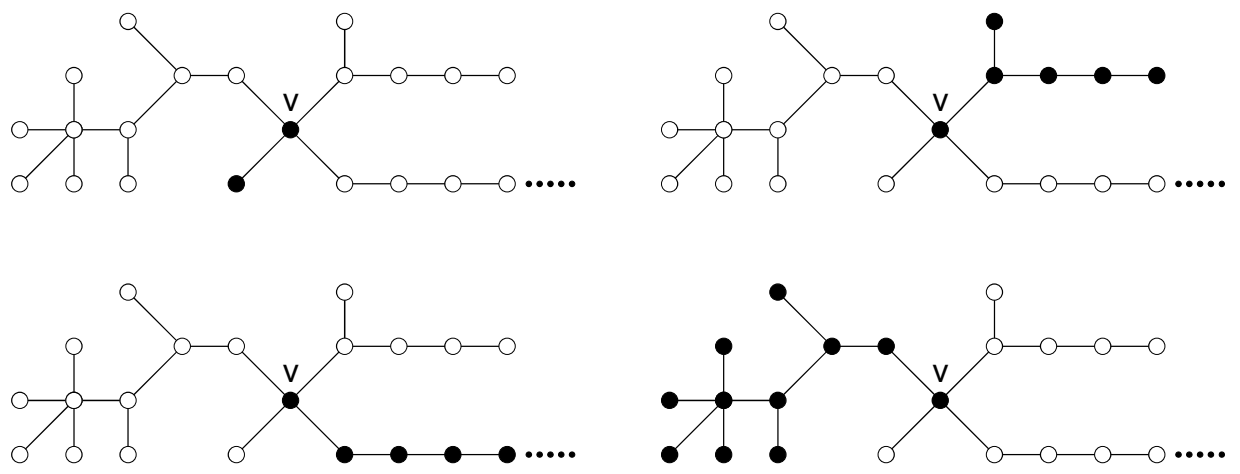

Figure 7: Branches of an infinite tree $T$ at a vertex $v$ with $\operatorname{deg}(v)=4$ and $P_{T}(v)=2$. At the left, the branch paths.

Theorem 4. If $T$ is an infinite tree with maximum degree at least three and finite metric 
dimension, then

$$
\beta(T)=\sum_{\operatorname{deg}(v) \geq 3} \max \left\{P_{T}(v)-1,0\right\},
$$

and all metric basis can be obtained in the following way: for each vertex $v$ such that $P_{T}(v)=k \geq 2$, select $k-1$ vertices different from $v$ lying on distinct branch paths of $T$ at $v$.

Proof. Let $S$ be one of the sets described in the theorem. By Theorem 3 , the set $W=$ $\{v \in V(T): \operatorname{deg}(v) \geq 3\}$ is finite. Since $P_{T}(v) \geq 2$ implies $\operatorname{deg}(v) \geq 3$, the set $S$ is also finite.

A resolving set of $T$ has at least so many vertices as $S$, since for any $v \in W$ such that $P_{T}(v) \geq 2$, two vertices adjacent to $v$ of different branch paths of $T$ at $v$ are resolved only by vertices of those two branch paths and different from $v$. Observe also that the sets of vertices of two different branch paths of $T$ at vertices of degree at least three are disjoint. Therefore, $|S|=\sum_{\operatorname{deg}(v) \geq 3} \max \left\{P_{T}(v)-1,0\right\}$.

We claim that $S$ resolves $T$. Consider two distinct vertices $x, y \in V(T)$. Let $T^{\prime}$ be the graph induced by the set of vertices that lie in some path with endpoints in $W \cup S \cup$ $\{x, y\} \cup N(W)$, where $N(W)$ is the set of vertices adjacent to some vertex of $W$. Then, $T^{\prime}$ is a finite tree that contains $W \cup S$, for all $v \in W$ we have $P_{T}(v)=P_{T^{\prime}}(v)$, and each branch path of $T^{\prime}$ at $v$ is contained in a branch path of $T$ at $v$. Therefore, $S$ is a resolving set for $T^{\prime}$, and, consequently, $S$ resolves the pair $x, y$ in $T$.

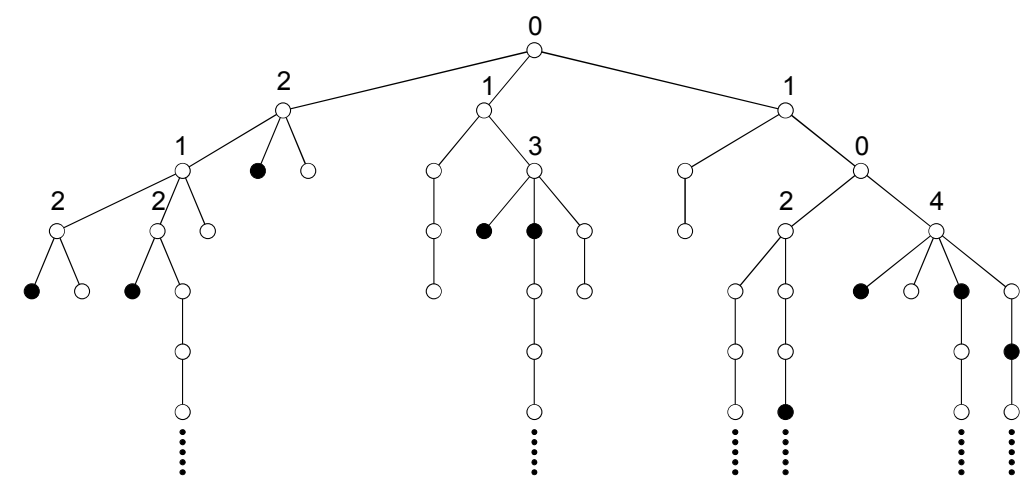

Figure 8: The parameter $P_{T}(v)$ for vertices of degree at least three. Black vertices form a metric basis of that infinite tree.

\section{Cartesian Products}

\subsection{Conditions for finite metric dimension}

The cartesian product of graphs $\mathrm{G}$ and $\mathrm{H}$, denoted by $G \square H$, is the graph with vertex set $V(G) \times V(H)=\{(a, v): a \in V(G), v \in V(H)\}$, where $(a, v)$ is adjacent to $(b, w)$ whenever $a=b$ and $v, w \in E(H)$, or $v=w$ and $a, b \in E(G)$. Observe that if $G$ and $H$ are connected, then $G \square H$ is connected. In particular, $d_{G \square H}((a, v),(b, w))=d_{G}(a, b)+d_{H}(v, w)$. A 
number of interesting examples can be viewed as the cartesian product of two graphs. For example, the two dimensional infinite grid is the graph $P_{2 \infty} \square P_{2 \infty}$.

Since the cartesian product of two infinite graphs has always infinite metric rays with pairwise disjoint vertex sets, it follows from Theorem 2 that:

Corollary 2. Given two infinite graphs $G$ and $H$, then $\beta(G \square H)=\infty$.

Given a subset $S$ of vertices in $G \square H$, its projection onto $G$ is the set of vertices $x \in V(G)$ for which there exists a vertex $u \in V(H)$ such that $(x, u) \in S$. Similarly is defined the projection of $S$ onto $H$. Some results obtained for finite graphs in [3] can be extended to infinite graphs.

Proposition 3. Let $G, H$ be finite or infinite graphs. If $S$ is a resolving set of $G \square H$, then the projection of $S$ onto $G$ (resp. onto $H$ ) is a resolving set of $G$ (resp. of $H$ ) and, consequently, $\beta(G \square H) \geq \max \{\beta(G), \beta(H)\}$.

Corollary 3. If $G$ is an infinite graph with infinite metric dimension, then for any graph $H$ we have $\beta(G \square H)=\infty$.

Another result that can be extended to infinite graphs is the following:

Theorem 5. If $G$ is an infinite graph with finite metric dimension and $H$ is a finite graph with at least two vertices, then the metric dimension of $G \square H$ is finite and $\beta(G \square H) \leq$ $\beta(G)+\psi(H)-1$

We do not include the proofs of Proposition 3 and Theorem 5 , since the proofs given in [3] also hold for infinite vertex sets.

We summarize the preceding results in Table 1.

\begin{tabular}{|cc|c|}
\hline$|V(G)|$ & $|V(H)|$ & $\beta(G \square H)$ \\
\hline$<\infty$ & $<\infty$ & $<\infty$ \\
\hline$=\infty$ & $=\infty$ & $=\infty$ \\
\hline$=\infty$ & $<\infty$ & $\begin{cases}<\infty & \text { if } \beta(G)<\infty, \\
=\infty & \text { if } \beta(G)=\infty . \\
\hline\end{cases}$
\end{tabular}

Table 1: Possibilities for the metric dimension of the cartesian product of graphs.

\subsection{Metric dimension of $P_{\infty} \square H$ and $P_{2 \infty} \square H$}

In this section, we study the metric dimension of the cartesian product of the infinite graphs $P_{\infty}$ and $P_{2 \infty}$ by finite graphs. In particular we determine the metric dimension of $P_{\infty} \square H$ and $P_{2 \infty} \square H$ when $H$ is a path, a cycle or a complete graph. Proposition 3 gives us a lower bound of $\beta(G \square H)$ in terms of $\beta(H)$. It is known that the metric dimension of paths, cycles and complete graphs of order $n \geq 3$ is respectively 1,2 and $n-1$ (see [4, 10]). Moreover, a vertex of degree 1 is a basis of a path; any two no antipodal vertices of a cycle form a basis of a cycle, and a basis of a complete graph is formed by any $n-1$ vertices of the graph. On the other hand, Theorem 5 gives an upper bound of $\beta(G \square H)$ in terms of $\psi(H)$. This parameter is determined in [3] for several families of graphs as paths $P_{n}$, 
cycles $C_{n}$ and complete graphs $K_{n}$ of order $n$. Concretely, $\psi\left(P_{n}\right)=2$, if $n \geq 2 ; \psi\left(C_{n}\right)=2$, if $n \geq 3$ odd; $\psi\left(C_{n}\right)=3$, if $n \geq 4$ even; and $\psi\left(K_{n}\right)=n-1$, if $n \geq 3$. In the rest of the paper we suppose that $V\left(P_{n}\right)=V\left(C_{n}\right)=V\left(K_{n}\right)=\{0,1,2, \ldots, n-1\}, V\left(P_{\infty}\right)=\mathbb{N}$ and $V\left(P_{2 \infty}\right)=\mathbb{Z}$. Two vertices $i, j$ are adjacent in $P_{n}$ or $P_{\infty}$ or $P_{2 \infty}$ if and only if $|j-i|=1$. Two vertices $0 \leq i \leq j \leq n-1$ are adjacent in $C_{n}$ if and only if $j-i=1$ or $j-i=n-1$, and any two distinct vertices of $K_{n}$ are adjacent.

\begin{tabular}{|c|cccc|}
\hline$G$ & $P_{n}$ & $C_{n}, n$ odd & $C_{n}, n$ even & $K_{n}$ \\
\hline$\beta(G)$ & 1 & 2 & 2 & $n-1$ \\
$\psi(G)$ & 2 & 2 & 3 & $n-1$ \\
\hline
\end{tabular}

Table 2: Values of $\beta(G)$ and $\psi(G)$ of paths, cycles and cliques of order $n \geq 3$.

Lemma 7. If $H$ is a graph, then $\beta\left(P_{2 \infty} \square H\right)=2$ if and only if $H$ is the trivial graph, $K_{1}$.

Proof. $(\Leftarrow)$ The graph $P_{2 \infty} \square K_{1}$ is $P_{2 \infty}$ with metric dimension 2 .

$(\Rightarrow)$ Suppose now that $H$ is a non trivial graph $H$ and $S=\{(i, u),(j, v)\}, i \leq j$, is a set with two different vertices of $P_{2 \infty} \square H$. We claim that $S$ does not resolve $P_{2 \infty} \square H$. By Proposition 3, if $i=j$, then the projection of $S$ onto $P_{2 \infty}$ is not a resolving set of $P_{2 \infty}$. Now suppose that $i \neq j$.

Case $u=v$. Let $w$ be a vertex adjacent to $u$ in $H$, that exists because $H$ is a non trivial graph. Then $d_{P_{2 \infty} \square H}((i-1, u),(i, u))=1=d_{H}(w, u)=d_{P_{2 \infty} \square H}((i, w),(i, u))$ and $d_{P_{2 \infty} \square H}((i-1, u),(j, u))=d_{P_{2 \infty}}(i-1, j)=d_{P_{2 \infty}}(i, j)+1=d_{P_{2 \infty}}(i, j)+d_{H}(w, u)=$ $d_{P_{2 \infty} \square H}((i, w),(j, u))$. Hence, $S$ does not resolve the vertices $(i-1, u)$ and $(i, w)$.

Case $u \neq v$. Let $w$ be a vertex of $H$ adjacent to $u$ and lying in a shortest $u-v$ path. Then $d_{P_{2 \infty} \square H}((i+1, u),(i, u))=1=d_{H}(w, u)=d_{P_{2 \infty} \square H}((i, w),(i, u))$ and $d_{P_{2 \infty} \square H}((i+$ $1, u),(j, v))=d_{P_{2 \infty}}(i+1, j)+d_{H}(u, v)=d_{P_{2 \infty}}(i, j)-1+d_{H}(u, v)=d_{P_{2 \infty}}(i, j)+d_{H}(w, v)=$ $d_{P_{2 \infty} \square H}((i, w),(j, v))$. Hence, $S$ does not resolve the vertices $(i+1, u)$ and $(i, w)$.

Lemma 8. If $H$ is a graph and $S \subseteq\{0\} \times V(H)$ is a resolving set of $P_{\infty} \square H$, then, for any $u \in V(H), S^{\prime}=S \cup\{(1, u)\}$ is a resolving set of $P_{2 \infty} \square H$.

Proof. Let $x=(i, v)$ and $y=(j, w)$ be two distinct vertices of $P_{2 \infty} \square H$. If $i, j \geq 0, S$ resolves $x$ and $y$. By symmetry, $S$ resolves $x$ and $y$ if $i, j \leq 0$.

Suppose now that $i>0$ and $j<0$. Since $r((j, w) \mid S)=r((-j, w) \mid S)$ and $S$ is a resolving set for $P_{\infty} \square H, S$ resolves $x$ and $y$ if $i \neq-j$ or $v \neq w$. Finally, for $i=-j>0$ and $v=w$ we have $d_{P_{2 \infty} \square H}(x,(1, u))=d_{P_{2 \infty} \square H}((i, v),(1, u))=i-1+d_{H}(v, u) \neq i+1+d_{H}(v, u)=$ $d_{P_{2 \infty} \square H}((-i, v),(1, u))=d_{P_{2 \infty} \square H}((j, w),(1, u))=d_{P_{2 \infty} \square H}(y,(1, u))$. Hence, $(1, u)$ resolves $x$ and $y$, and consequently $S^{\prime}$ is a resolving set for $P_{2 \infty} \square H$.

We determine now the metric dimension and a metric basis of $G \square P_{n}$, when $G$ is $P_{\infty}$ or $P_{2 \infty}$ and $n \geq 2$. We have in that case

$$
d_{G \square P_{n}}\left((i, j),\left(i^{\prime}, j^{\prime}\right)\right)=d_{G}(i, j)+d_{P_{n}}\left(i^{\prime}, j^{\prime}\right)=\left|i^{\prime}-i\right|+\left|j^{\prime}-j\right| .
$$

Proposition 4. For all $n \geq 2, \beta\left(P_{\infty} \square P_{n}\right)=2$ and $S=\{(0,0),(0, n-1)\}$ is a metric basis of $P_{\infty} \square P_{n}$. 
Proof. By Theorem 5, $\beta\left(P_{\infty} \square P_{n}\right) \leq \beta\left(P_{\infty}\right)+\psi\left(P_{n}\right)-1=1+2-1=2$ if $n \geq 2$. Since the infinite graph $P_{\infty} \square P_{n}$ is not the one-way infinite path for $n \geq 2$, by Proposition 1 we have $\beta\left(P_{\infty} \square P_{n}\right)=2$ for all $n \geq 2$.

Consider now two different vertices $(i, j)$ and $\left(i^{\prime}, j^{\prime}\right)$ in $P_{\infty} \square P_{n} . S$ is a resolving set of the subgraph isomorphic to $P_{m} \square P_{n}$ that contains $S,(i, j)$ and $\left(i^{\prime}, j^{\prime}\right)$, where $m=\max \left\{i, i^{\prime}\right\}+1$ (see [10]). Then, $S$ resolves the pair $(i, j)$ and $\left(i^{\prime}, j^{\prime}\right)$ in $P_{\infty} \square P_{n}$.

Proposition 5. For all $n \geq 2, \beta\left(P_{2 \infty} \square P_{n}\right)=3$ and $S=\{(0,0),(0, n-1),(1,0)\}$ is a metric basis of $P_{2 \infty} \square P_{n}$.

Proof. By Theorem 5 and Proposition $3,2=\beta\left(P_{2 \infty}\right) \leq \beta\left(P_{2 \infty} \square P_{n}\right) \leq \beta\left(P_{2 \infty}\right)+\psi\left(P_{n}\right)-$ $1=2+2-1=3$. By Lemma 7, $\beta\left(P_{2 \infty} \square P_{n}\right) \neq 2$. Hence, $\beta\left(P_{2 \infty} \square P_{n}\right)=3$. See Figure 15 .

By Proposition 4 and Lemma $8, S$ is a metric basis of $P_{2 \infty} \square P_{n}$.

For determining the metric dimension and a metric basis of $G \square C_{n}$, when $G$ is $P_{\infty}$ or $P_{2 \infty}$ and $n \geq 3$, observe that

$$
d_{G \square C_{n}}\left((i, j),\left(i^{\prime}, j^{\prime}\right)\right)=d_{G}(i, j)+d_{C_{n}}\left(i^{\prime}, j^{\prime}\right)=\left|i^{\prime}-i\right|+\min \left\{\left|j^{\prime}-j\right|, n-\left|j^{\prime}-j\right|\right\} .
$$

Proposition 6. For all $n \geq 3$, the metric dimension of $P_{\infty} \square C_{n}$ is

$$
\beta\left(P_{\infty} \square C_{n}\right)=\left\{\begin{array}{l}
2 \quad \text { if } n \text { is odd, } \\
3 \quad \text { if } n \text { is even, }
\end{array}\right.
$$

and $S_{1}=\left\{(0,0),\left(0, \frac{n-1}{2}\right)\right\}$ is a metric basis, if $n$ is odd, and $S_{2}=\left\{(0,0),\left(0, \frac{n}{2}\right),(0,1)\right\}$ is a metric basis, if $n$ is even.

Proof. By Theorem 5 and Proposition $3,2=\beta\left(C_{n}\right) \leq \beta\left(P_{\infty} \square C_{n}\right) \leq \beta\left(P_{\infty}\right)+\psi\left(C_{n}\right)-1=$ $\psi\left(C_{n}\right)$. Therefore, $\beta\left(P_{\infty} \square C_{n}\right)$ is 2 , if $n$ is odd, and 2 or 3 , if $n$ is even.

Suppose now that $n$ is even and $W=\left\{(i, j),\left(i^{\prime}, j^{\prime}\right)\right\}$ is a set with two different vertices of $P_{\infty} \square C_{n}$. If $W$ is a resolving set of $P_{\infty} \square C_{n}$, the projection of $W$ onto $P_{\infty}$ is a resolving set of $P_{\infty}$ and the projection onto $C_{n}$ is a resolving set of $C_{n}$. Therefore, $i=i^{\prime}=0$ or $i \neq i^{\prime}$, and $j, j^{\prime}$ are different vertices not antipodal in $C_{n}$. By symmetry, we may assume that $0=j<j^{\prime}<\frac{n}{2}$. If $i=i^{\prime}=0, W$ does not resolve the pair of vertices $\left(0, j^{\prime}+1\right)$ and $\left(1, j^{\prime}\right)$ (see Figure 9, left). If $i \neq i^{\prime}$, we may assume by symmetry that $0 \leq i<i^{\prime}$. Then $W$ does not resolve the pair of vertices $(i, 1)$ and $(i+1,0)$ (see Figure 9, right). In any case, we have a contradiction. Hence $\beta\left(P_{\infty} \square C_{n}\right)=3$ for $n$ even.

Now, for $n=2 k+1$ odd, consider the set $S_{1}=\{(0,0),(0, k)\}$. Then,

$$
r\left((0, j) \mid S_{1}\right)= \begin{cases}(j, k-j) & \text { if } 0 \leq j \leq k, \\ (2 k+1-j, j-k) & \text { if } k+1 \leq j \leq 2 k .\end{cases}
$$

Observe that the sum of the two coordinates is $k$ in the first case and $k+1$ otherwise. For $i>0$ we have $r\left((i, j) \mid S_{1}\right)=r\left((0, j) \mid S_{1}\right)+(i, i)$. Now, the sum of the two coordinates is $2 i+k$ if $0 \leq j \leq k$ and $2 i+k+1$ if $k+1 \leq j \leq 2 k$. Suppose that the vertices $x=(i, j)$ and $y=\left(i^{\prime}, j^{\prime}\right)$, where $0 \leq i \leq i^{\prime}$, satisfy $r\left((i, j) \mid S_{1}\right)=r\left(\left(i^{\prime}, j^{\prime}\right) \mid S_{1}\right)$. This implies $r\left((0, j) \mid S_{1}\right)=r\left(\left(i^{\prime}-i, j^{\prime}\right) \mid S_{1}\right)$. But this is not possible when $i^{\prime}-i \neq 0$, since the sum of the two coordinates of $r\left((0, j) \mid S_{1}\right)$ is $k$ or $k+1$, and the sum of the two coordinates of $r\left(\left(i^{\prime}-i, j^{\prime}\right) \mid S_{1}\right)$ is $2\left(i^{\prime}-i\right)+k$ or $2\left(i^{\prime}-i\right)+k+1$. Then $i^{\prime}=i$ and, from (1), we have $j=j^{\prime}$. Thus, $S_{1}$ resolves $P_{\infty} \square C_{n}$ if $n$ is odd. 

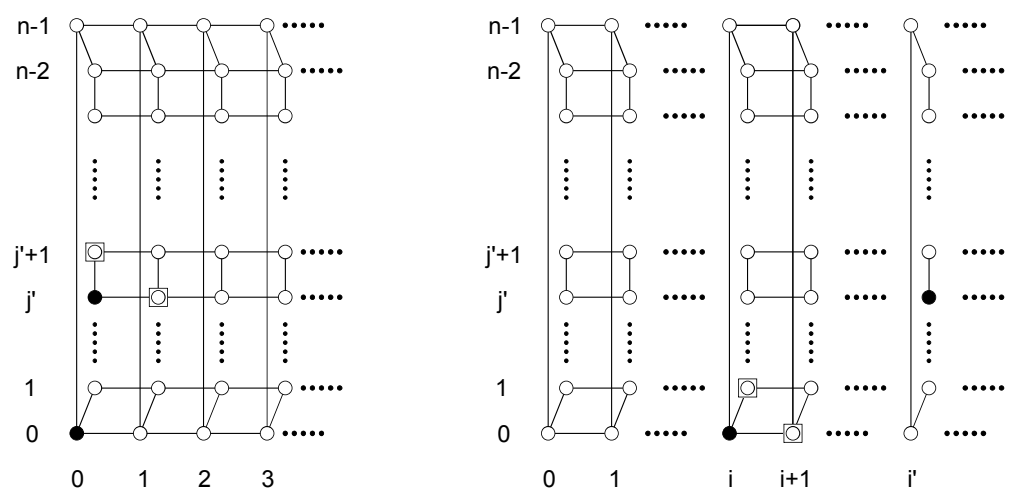

Figure 9: For $n$ even, black vertices do not resolve squared vertices if $0<j^{\prime}<\frac{n}{2}$ (left) and if $0 \leq i<i^{\prime}, 0<j^{\prime}<\frac{n}{2}$ (right).

If $n=2 k$ even, consider the set $S_{2}=\{(0,0),(0, k),(0,1)\}$. Then,

$$
r\left((0, j) \mid S_{2}\right)= \begin{cases}(0, k, 1) & \text { if } j=0 \\ (j, k-j, j-1) & \text { if } 1 \leq j \leq k \\ (2 k-j, j-k, 2 k-j+1) & \text { if } k+1 \leq j \leq 2 k-1\end{cases}
$$

Observe that the sum of the first two coordinates is $k$. For $i>0$ we have $r\left((i, j) \mid S_{2}\right)=$ $r\left((0, j) \mid S_{2}\right)+(i, i, i)$, and the sum of the first two coordinates is $2 i+k$.

Suppose that the vertices $x=(i, j)$ and $y=\left(i^{\prime}, j^{\prime}\right)$, where $0 \leq i \leq i^{\prime}$, satisfy $r\left((i, j) \mid S_{2}\right)=r\left(\left(i^{\prime}, j^{\prime}\right) \mid S_{2}\right)$. This implies $r\left((0, j) \mid S_{2}\right)=r\left(\left(i^{\prime}-i, j^{\prime}\right) \mid S_{2}\right)$. But this is not possible when $i^{\prime}-i \neq 0$, since the sum of the first two coordinates of $r\left((0, j) \mid S_{2}\right)$ is $k$, and the sum of the first two coordinates of $r\left(\left(i^{\prime}-i, j^{\prime}\right) \mid S_{2}\right)$ is $2\left(i^{\prime}-i\right)+k$. Then $i^{\prime}=i$ and, from (2), we have that $j=j^{\prime}$. Thus, $S_{2}$ resolves $P_{\infty} \square C_{n}$ if $n$ is even.

Proposition 7. For all $n \geq 3$,

$$
\beta\left(P_{2 \infty} \square C_{n}\right)= \begin{cases}3 & \text { if } n \text { is odd, } \\ 4 & \text { if } n \text { is even. }\end{cases}
$$

and a metric basis of $P_{2 \infty} \square C_{n}$ is $S_{1}=\left\{(0,0),\left(0, \frac{n-1}{2}\right),(1,0)\right\}$, if $n$ is odd, and $S_{2}=$ $\left\{(0,0),\left(0, \frac{n}{2}\right),(0,1),(1,0)\right\}$, if $n$ is even.

Proof. By Theorem 5 and Proposition $3,2=\beta\left(C_{n}\right) \leq \beta\left(P_{2 \infty} \square C_{n}\right) \leq \beta\left(P_{2 \infty}+\psi\left(C_{n}\right)-1=\right.$ $\psi\left(C_{n}\right)+1$. By Lemma 7, $\beta\left(P_{\infty} \square C_{n}\right) \neq 2$. Therefore, $\beta\left(P_{2 \infty} \square C_{n}\right)=3$, if $n$ is odd, and $\beta\left(P_{\infty} \square C_{n}\right)$ is 3 or 4 , if $n$ is even. On the other hand, Proposition 6 and Lemma 8 imply that $S_{1}$ is a metric basis of $P_{\infty} \square C_{n}$ for $n$ odd.

Suppose now that $n$ is even and let $S=\left\{(i, j),\left(i^{\prime}, j^{\prime}\right),\left(i^{\prime \prime}, j^{\prime \prime}\right)\right\}$ be a set vertices of $G=P_{2 \infty} \square C_{n}$ of cardinality 3 . We claim that $S$ does not resolve $G$.

If $\left|\left\{j, j^{\prime}, j^{\prime \prime}\right\}\right|=1$ or $\left\{j, j^{\prime}, j^{\prime \prime}\right\}$ is a set of two antipodal vertices of $C_{n}$, then $S$ does not resolve $P_{\infty} \square C_{n}$ by Proposition 3 .

If $\left\{j, j^{\prime}, j^{\prime \prime}\right\}$ is a set of two no antipodal vertices of $C_{n}$, we may assume by symmetry that $j=j^{\prime}=0,0<j^{\prime \prime}<\frac{n}{2}$ and $\min \left\{i, i^{\prime}, i^{\prime \prime}\right\}=0$. Then $S$ does not resolve $(-1,0)$ and $(0, n-1)$ (see Figure 10, left). 

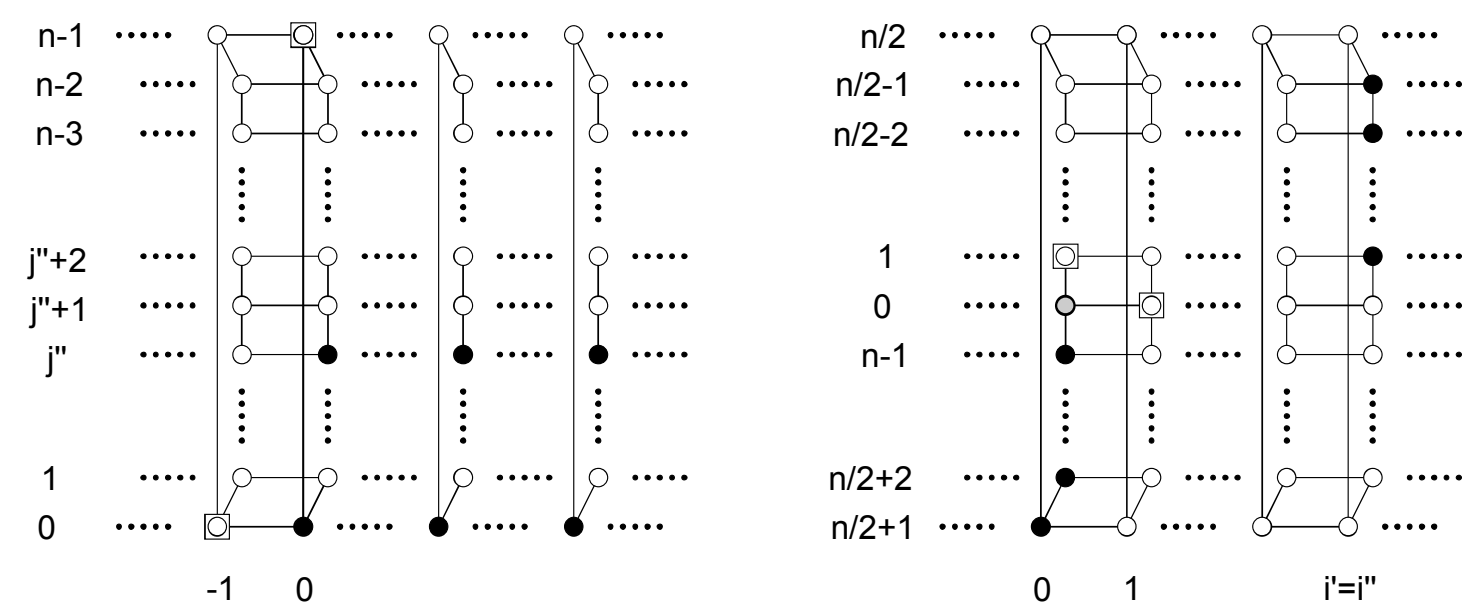

Figure 10: For $n$ even, three black vertices of different columns do not resolve squared vertices if $0<j^{\prime \prime}<\frac{n}{2}$ (left) and vertex $(0,0)$ together with two black vertices of different columns do not resolve squared vertices (right).

If $\left|\left\{j, j^{\prime}, j^{\prime \prime}\right\}\right|=3$, at least one vertex is not the antipodal of the remaining two vertices. In any case, we may assume without lose of generalization that $j=0,0<j^{\prime}<n / 2$ and $n / 2<j^{\prime \prime} \leq n-1$. Now, if $\left|\left\{i, i^{\prime}, i^{\prime \prime}\right\}\right|=1, S$ is not a resolving set by Proposition 3 . If $\left|\left\{i, i^{\prime}, i^{\prime \prime}\right\}\right|=2$, we may assume by symmetry that all the cases are analogous to (1) $i=i^{\prime \prime}=0$ and $i^{\prime}>0$, or (2) $i=0$ and $i^{\prime}=i^{\prime \prime}>0$. In the first case, $S$ does not resolve $(0,1)$ and $(1,0)$ (see Figure 10 , right). In the second case, $S$ does not resolve $\left(i^{\prime}, j^{\prime}+1\right)$ and $\left(i^{\prime}+1, j^{\prime}\right)$ if $j^{\prime \prime}-j^{\prime}>\frac{n}{2} ; S$ does not resolve $\left(i^{\prime}-1, j^{\prime}+1\right)$ and $\left(i^{\prime}+1, j^{\prime}-1\right)$ if $j^{\prime \prime}-j^{\prime}=\frac{n}{2}$; and $S$ does not resolve $\left(i^{\prime}-1, j^{\prime}\right)$ and $\left(i^{\prime}, j^{\prime}-1\right)$ if $j^{\prime \prime}-j^{\prime}<\frac{n}{2}$ (see Figure 11).

Finally, if $\left|\left\{i, i^{\prime}, i^{\prime \prime}\right\}\right|=3$, by symmetry all the cases are analogous to (1) $i^{\prime \prime}<i=0<i^{\prime}$ or (2) $i<i^{\prime \prime}=0<i^{\prime}$. In both cases $S$ does not resolve $(0,1)$ and $(1,0)$ (see Figure 12).

Thus, $S$ does not resolve $P_{2 \infty} \square C_{n}$ when $n$ is even, implying that the metric dimension is 4. By Proposition 6 and Lemma 8, $S_{2}$ is a metric basis of $P_{2 \infty} \square C_{n}$.

Finally, we give the metric dimension and a metric basis of $G \square K_{n}$, when $G$ is $P_{\infty}$ or $P_{2 \infty}$ and $n \geq 4$. We have in that case

$$
d_{G \square K_{n}}\left((i, j),\left(i^{\prime}, j^{\prime}\right)\right)= \begin{cases}\left|i^{\prime}-i\right|, & \text { if } j=j^{\prime}, \\ \left|i^{\prime}-i\right|+1, & \text { if } j \neq j^{\prime} .\end{cases}
$$

Proposition 8. For all $n \geq 4, \beta\left(P_{\infty} \square K_{n}\right)=n-1$ and $S=\{(0,0),(0,1), \ldots,(0, n-2)\}$ is a metric basis of $P_{\infty} \square K_{n}$.

Proof. By Theorem 5 and Proposition 3, $n-1=\beta\left(K_{n}\right) \leq \beta\left(P_{\infty} \square K_{n}\right) \leq \beta\left(P_{\infty}\right)+\psi\left(K_{n}\right)-$ $1=\psi\left(K_{n}\right)=n-1$. Hence, $\beta\left(P_{\infty} \square K_{n}\right)=n-1$.

Consider now a pair of two distinct vertices, $x=(i, j), y=\left(i^{\prime}, j^{\prime}\right)$. If $i=i^{\prime}$, the vertex $(0, h)$ resolves $x$ and $y$, where $h=\min \left\{j, j^{\prime}\right\} \in[0, n-2]$. If $i \neq i^{\prime}$, the vertex $(0, h)$ resolves $x$ and $y$, where $h$ is any integer $h \in[0, n-2]$ such that $h \neq j, j^{\prime}$. Thus, $S$ is a metric basis of $P_{\infty} \square K_{n}$. 


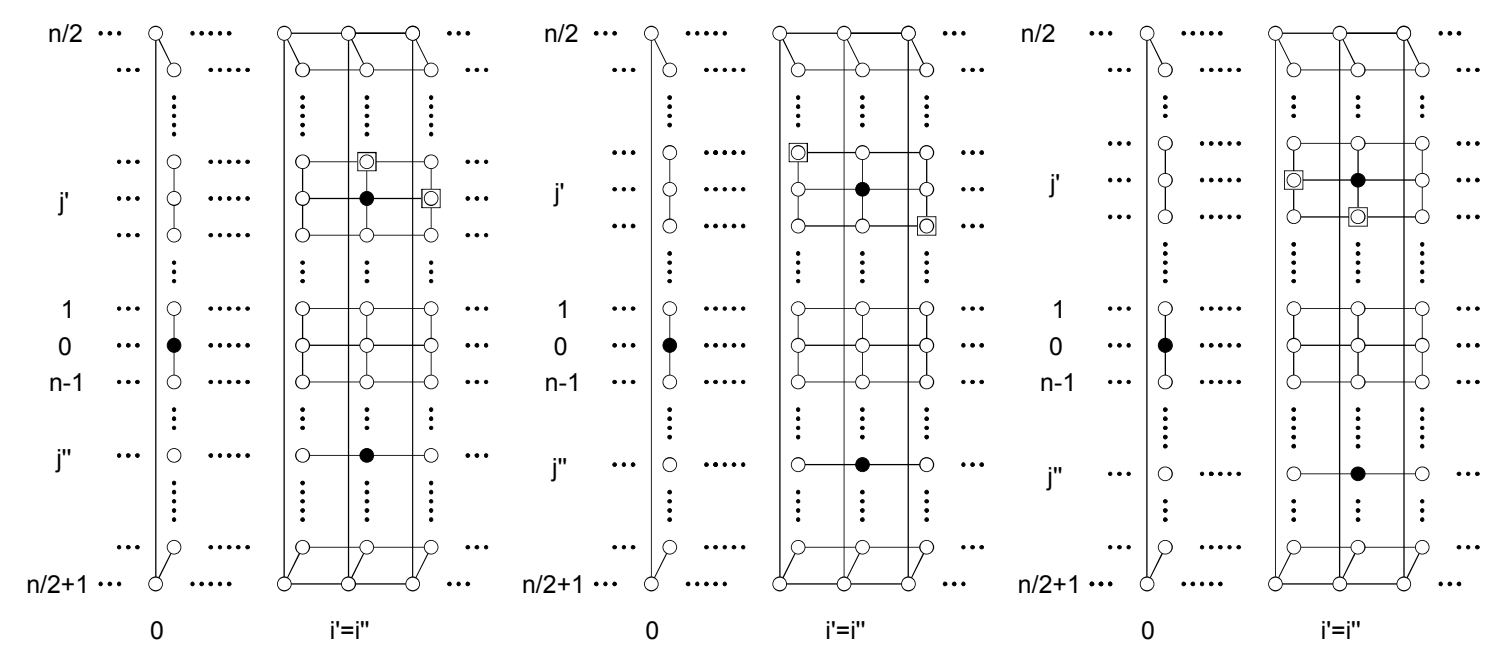

Figure 11: Black vertices do not resolve squared vertices if $j^{\prime \prime}-j^{\prime}>n / 2$ (left), if $j^{\prime \prime}-j^{\prime}=$ $n / 2$ (middle), and if $j^{\prime \prime}-j^{\prime}<n / 2$ (right).
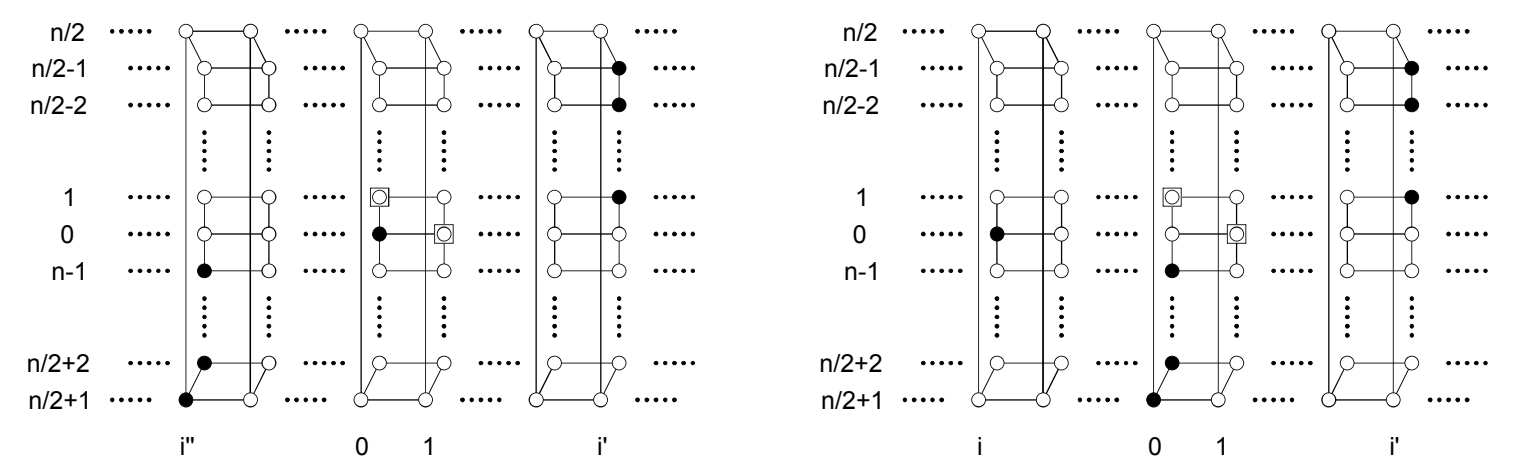

Figure 12: Three black vertices of different columns do not resolve squared vertices.

Proposition 9. For all $n \geq 4, \beta\left(P_{2 \infty} \square K_{n}\right)=n-1$ and $S=\{(0,0),(1,1), \ldots,(n-2, n-2)\}$ is a metric basis of $P_{2 \infty} \square K_{n}$.

Proof. By Theorem 5 and Proposition 3, $n-1=\beta\left(K_{n}\right) \leq \beta\left(P_{2 \infty} \square K_{n}\right) \leq \beta\left(P_{2 \infty}\right)+$ $\psi\left(K_{n}\right)-1=\psi\left(K_{n}\right)+1=n$. Hence, $\beta\left(P_{\infty} \square K_{n}\right)$ is $n-1$ or $n$.

We show next that $S$ is a metric basis of $P_{2 \infty} \square K_{n}$. Let $x=(i, j), y=\left(i^{\prime}, j^{\prime}\right)$ be two different vertices of $P_{2 \infty} \square K_{n}$. If $i=i^{\prime}$, the vertex $(h, h)$ resolves $x$ and $y$, where $h=\min \left\{j, j^{\prime}\right\} \in[0, n-2]$. Suppose now that $i \neq i^{\prime}$. Consider $k \in[0, n-2]$ such that $k \neq j, j^{\prime}$ and with the additional condition $k \neq \frac{i+i^{\prime}}{2}$ if the distance $d_{P_{2 \infty}}\left(i, i^{\prime}\right)$ is even.

Observe that such a $k$ exists except for the case $n=4$ and $\left\{j, j^{\prime}, \frac{i+i^{\prime}}{2}\right\}=\{0,1,2\}$. In any other case, In any other case, $|k-i| \neq\left|k-i^{\prime}\right|$. Therefore, $d_{P_{2 \infty} \square K_{n}}((i, j),(k, k))=$ $|k-i|+1 \neq\left|k-i^{\prime}\right|+1=d_{P_{2 \infty} \square K_{n}}\left(\left(i^{\prime}, j^{\prime}\right),(k, k)\right)$. Hence, $(k, k) \in S$ resolves $x$ and $y$ (see Figure 13.

It only remains to prove that $S=\{(0,0),(1,1),(2,2)\}$ resolves $x$ and $y$ in $P_{2 \infty} \square K_{4}$ when $\left\{j, j^{\prime}, \frac{i+i^{\prime}}{2}\right\}=\{0,1,2\}$. 


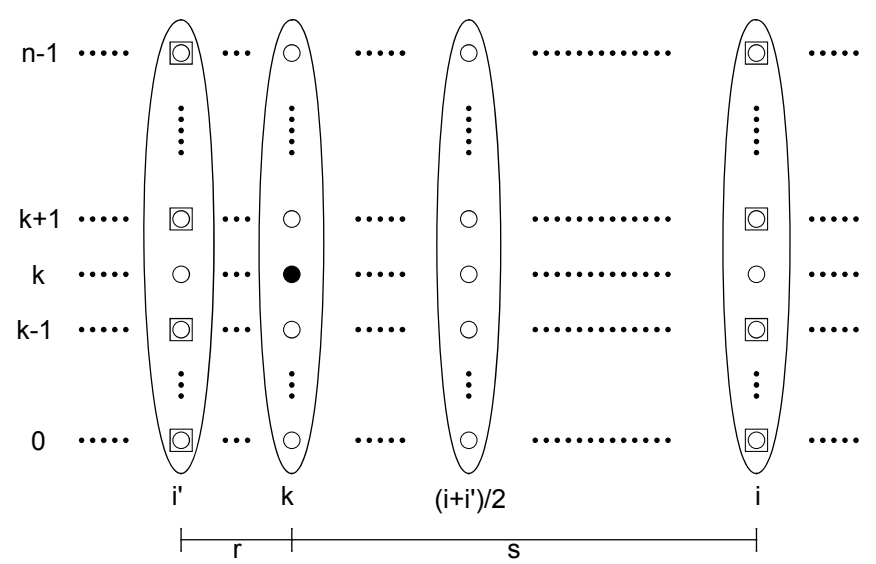

Figure 13: Black vertex resolves two squared vertices of different columns if $r \neq s$.

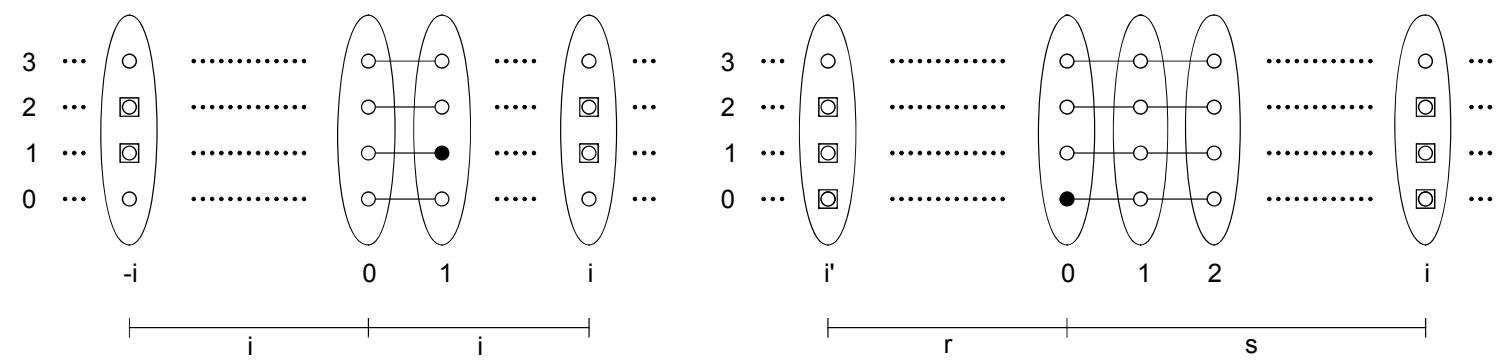

Figure 14: Black vertex resolves two squared vertices of different columns if $i^{\prime}=-i$ (left) and if $\left(i+i^{\prime}\right) / 2 \in\{0,1\}$, and therefore $r \leq s-2$ (right).

Case $\frac{i+i^{\prime}}{2}=0$. This implies $i^{\prime}=-i \neq 0$ and $\left\{j, j^{\prime}\right\}=\{1,2\}$. We may assume $i>0$. Then, $d_{P_{2 \infty} \square K_{4}}((1,1),(i, j))=(i-1)+d_{K_{4}}(1, j) \leq(i-1)+1=i<i+1=d_{P_{2 \infty}}(1,-i)=$ $d_{P_{2 \infty}}\left(1, i^{\prime}\right) \leq d_{P_{2 \infty}}\left(1, i^{\prime}\right)+d_{K_{4}}\left(1, j^{\prime}\right)=d_{P_{2 \infty} \square K_{4}}\left((1,1),\left(i^{\prime}, j^{\prime}\right)\right)$. Therefore, $(1,1)$ resolves $x$ and $y$ (see Figure 14 , left).

Case $\frac{i+i^{\prime}}{2} \in\{1,2\}$. Assume $i>i^{\prime}$. Then, $i \geq 2$ and $i^{\prime} \leq 0$, if $\frac{i+i^{\prime}}{2}=1$, or $i^{\prime} \leq 1$, if $\frac{i+i^{\prime}}{2}=2$. For $i^{\prime} \leq 0, d_{P_{2 \infty} \square K_{4}}((0,0),(i, j))=i+d_{K_{4}}(0, j) \geq i>-i^{\prime}+1 \geq-i^{\prime}+d_{K_{4}}\left(0, j^{\prime}\right)=$ $d_{P_{2 \infty} \square K_{4}}\left((0,0),\left(i^{\prime}, j^{\prime}\right)\right)$. Therefore, $(0,0)$ resolves $x$ and $y$ (see Figure 14, right). Finally, the case $i^{\prime}=1$ is only possible for $\frac{i+i^{\prime}}{2}=2$, and therefore $i=3,\left\{j, j^{\prime}\right\}=\{0,1\}$. Then, $d_{P_{2 \infty} \square K_{4}}\left((0,0),\left(1, j^{\prime}\right)\right)=1+d_{K_{4}}\left(0, j^{\prime}\right) \leq 2<3=d_{P_{2 \infty}}(0,3) \leq d_{P_{2 \infty} \square K_{4}}((0,0),(3, j))$. Thus, $(0,0)$ resolves $x$ and $y$.

We summarize the results obtained in the preceding propositions in Table 3 and illustrate the given metric basis in Figures 15,16 and 17.

\begin{tabular}{|c|cccc|}
\hline$G \backslash H$ & $P_{n}, n \geq 2$ & $C_{n}, n \geq 3$ odd & $C_{n}, n \geq 4$ even & $K_{n}, n \geq 4$ \\
\hline$P_{\infty}$ & 2 & 2 & 3 & $n-1$ \\
$P_{2 \infty}$ & 3 & 3 & 4 & $n-1$ \\
\hline
\end{tabular}

Table 3: Metric dimension of $G \square H$ for some families of graphs. 

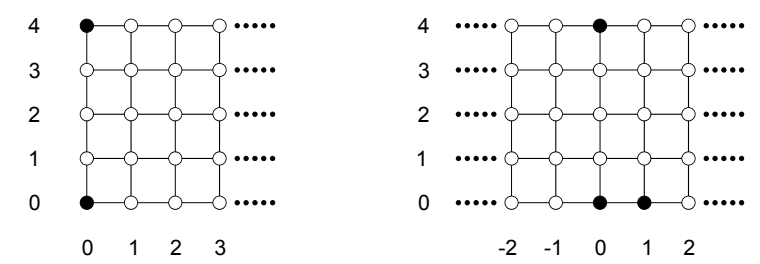

Figure 15: Black vertices form a metric basis of the graphs $P_{\infty} \square P_{5}$ (left) and $P_{2 \infty} \square P_{5}$ (right).
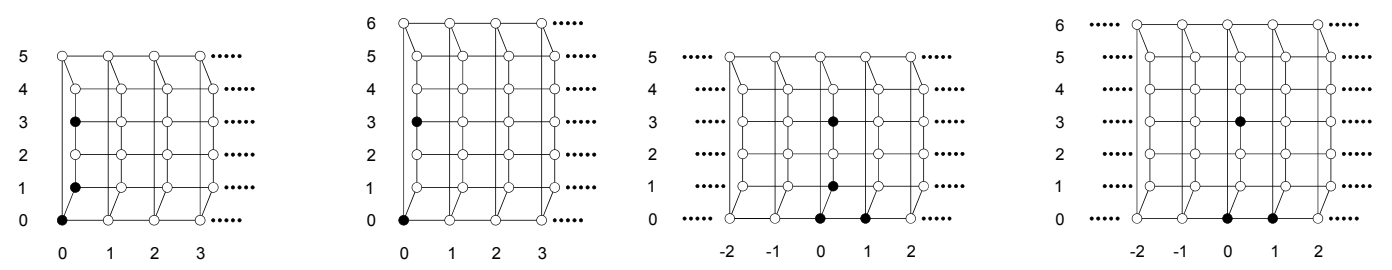

Figure 16: From left to right, black vertices form a metric basis of the graphs $P_{\infty} \square C_{6}$, $P_{\infty} \square C_{7}, P_{2 \infty} \square C_{6}$ and $P_{2 \infty} \square C_{7}$.
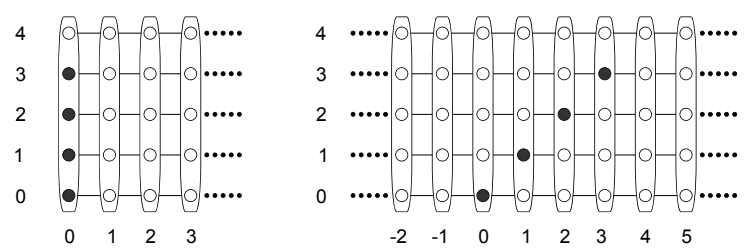

Figure 17: Black vertices form a metric basis of the graphs $P_{\infty} \square K_{5}$ (left) and $P_{2 \infty} \square K_{5}$ (right). The vertices of each column are pairwise adjacent.

\section{References}

[1] N. Alon, D. N. Kozlov, and V. H. Vu. The geometry of coinweighing problems. Proc. 37th Annual Symposium on Foundations of Computer Science (FOCS 96), 524-532. IEEE, 1996.

[2] Z. Beerliova, F. Eberhard, T. Erlebach, A. Hall, M. Hoffmann, M. Mihalak, and L. Shankar Ram. Network discovery and verification. Proc. 31st Workshop on Graph Theoretic Concepts in Computer Science (WG 05), in Lecture Notes in Comput. Sci. 3787:127-138, Springer, 2005

[3] J. Cáceres, C. Hernando, M. Mora, M. L. Puertas, I. M. Pelayo, C. Seara, and D. R. Wood. On the Metric Dimension of Cartesian Products of Graphs. SIAM Journal of Discrete Mathematics, 21(2):423-441, 2007.

[4] G. Chartrand, L. Eroh, M. A. Johnson, and O. R. Oellermann. Resolvability in graphs and the metric dimension of a graph. Discrete Appl. Math., 105(1-3):99-113, 2000.

[5] G. Chartrand and P. Zhang. The theory and applications of resolvability in graphs. A survey. Proc. 34th Southeastern International Conf. on Combinatorics, Graph Theory and Computing, in Congr. Numer. 160: 47-68, 2003. 
[6] R. Diestel (Ed.). Directions in Infinite Graphs and Combinatorics. Topics in Discrete Mathematics 3. Elsevier-North Holland, 1992.

[7] C. Hernando, M. Mora, I. M. Pelayo, C. Seara, and D. Wood. Extremal graph theory for metric dimension and diameter. Electronic Journal of Combinatorics, submitted. http://www . arxiv.org/math/0705.0938v1.

[8] V. Chvátal. Mastermind. Combinatorica, 3(3-4):325-329, 1983.

[9] D. König. Theory of Finite and Infinite Graphs. Birkhäuser, Boston, 1990.

[10] S. Khuller, B. Raghavachari, and A. Rosenfeld. Landmarks in graphs. Discrete Appl. Math., 70(3):217-229, 1996.

[11] F. Harary and R. Melter. On the metric dimension of a graph. Ars Combinatoria, 2:191195, 1976.

[12] C. St. J. A. Nash-Williams. Infinite graphs. A Survey. Journal of Combinatorial Theory, 3:286-301, 1967.

[13] A. Sebö and E. Tannier. On metric generators of graphs. Math. Oper. Res., 29(2):383393, 2004.

[14] B. Shanmukha, B. Sooryanarayana, and K. S. Harinath. Metric dimension of wheels. Far East J. Appl. Math., 8(3):217-229, 2002.

[15] P. Slater. Leaves of trees. Proc. 6th Southeastern Conf. on Combinatorics, Graph Theory, and Computing, in Congr. Numer., 14:549-559, 1975.

[16] C. Thomassen. Infinite Graphs. Further Selected Topics in Graph Theory, 129-160. Academic Press, London, 1983. 